\title{
Design, Modeling And Analysis Of A New Dual Input-Output Switched Capacitor Converter
}

\author{
Ainur Zhaikhan ${ }^{1}$, Vivekanandan Subburaj ${ }^{2}$, Debashisha Jena², Parthiban Perumal ${ }^{2}$, and Alexander Ruderman ${ }^{1}$ \\ ${ }^{1}$ Department of Electrical and Electronics Engineering, Nazarbayev University, Kazakhstan \\ ${ }^{2}$ Department of Electrical and Electronics Engineering, NIT Surathkal, INDIA
}

\begin{abstract}
A new dual-input and dual-output switched capacitor (SC) converter is designed to operate with two independent voltage sources that provides two different output voltages. The only converter generates 32 voltage conversion ratios (VCRs). The converter is portable to operate with one or two input sources alternatively and having the ability to vary 32 voltage ratios. An efficient low power SC converter is designed for input voltage of $1.5 \mathrm{~V}$ to $5 \mathrm{~V}$ that gives dual output voltages of $1 \mathrm{~V}$ to $10 \mathrm{~V}$. The designed converter can operate in both buck and boost modes. This SC converter has high drive capability of load current from $10 \mu \mathrm{A}$ to $25 \mathrm{~mA}$ that is adjusted by operating frequency. Modeling, analysis are performed to verify the dual output converter mathematically and also verified using PSIM simulations. The mathematical results and simulation results show excellent proof of newly designed converter.
\end{abstract}

Index Terms-DC/DC converter, SC converter, VCRs, dualinput, dual-output, multiphase, series-parallel.

\section{INTRODUCTION}

Portable electronics are the major development in electronic industries. Recent electronics equipment are made smaller and smaller simultaneously their efficiency is increasing a lot. Nowadays batteries are used to feed the power to the electronics devices, so battery gives the input to the integrated devices of the different parts of the circuits. But batteries can provide only a nominal DC voltage meanwhile integrated circuits require different DC voltages for the various parts of the circuit. Hence power management circuit is required. Many different converters are available on the market, hence switched capacitor converter is used among all kinds of converters. The main advantage of the switched capacitor (SC) converters is their smaller size, nonmagnetic components, and high efficiency [1]. In addition, by reconfiguring the topology we can generate maximum number of voltage conversion ratios (VCRs). Different topology was designed by researchers on SC converters. Authors in [2] generate 6 VCRs of Fibonacci fractions using 10 switches and 3 capacitors. Authors in [3] used 16 switches 5 capacitors to generate 21 Fibonacci fractions.

In SC converter design, two inputs are utilized for generating two different outputs. One of the inputs is renewable sources (solar, fuelcell, biomass etc.,) second input is battery. The main feature of proposed converter, can generate 32 VCRs by simply reconfiguring the converter switches. The selection of $32 \mathrm{VCRs}$ is based on the voltage specification of the low power system. Authors in [4] generate 11 VCRs with single output.

Some commercial products are available in market for different VCRs with usage of multiple SC converters. MAX integrated, implemented an IC for single input dual output SC converter is explained in [5]. Similarly Texas Instruments also developed SC converter with single input which is explained in [6]. Another example is power management IC with 20 regulated outputs, also developed by Texas Instruments [7]. The above commercial SC converters are operated using single input with multiple SC converters to generate maximum VCRs. To overcome the above problem, dual input and dual output SC converter is designed with single circuit.

In this work, 11 switches and 2 capacitors are used to generate 32 VCRs. The model of SC converter is required to validate the converter design mathematically. The modeling of the proposed converter follows average-current methodology. The converter is analysed for different VCRs and the modeling includes losses of capacitor and on switch resistance.

\section{Circuit Description}

The proposed SCC configuration is illustrated in Fig 1. It is basically a 2 capacitor series-parallel SC converter.

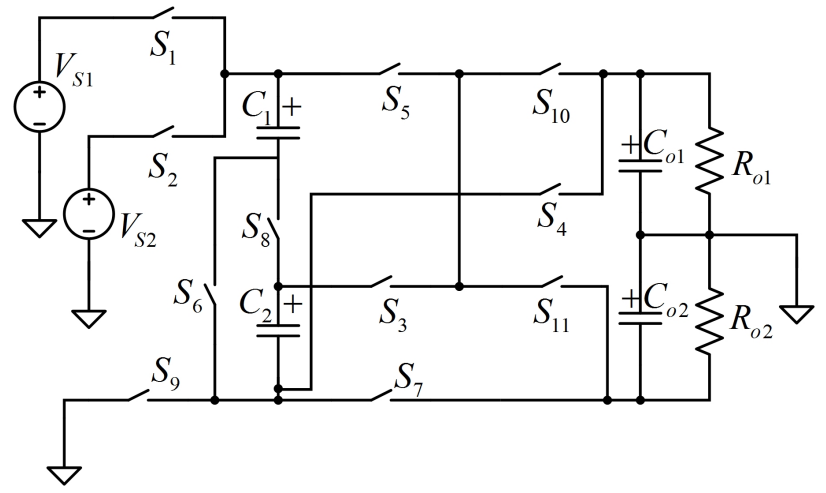

Fig. 1: Proposed dual input dual output SC converter

The proposed design SC converter generates 32 VCRs with limited switches and capacitors. In total 2 capacitors and 11 switches. The main advantage of the circuit is that both loads can appear in the same topology. So, it is possible to configure one of the output to certain voltage, and use this value to generate different VCR in the second output. 
TABLE I: 32 VCRs

\begin{tabular}{|c|c|c|c|c|c|c|c|c|c|c|c|c|}
\hline Multiple TR & $\phi$ & $S_{1}$ & $S_{2}$ & $S_{3}$ & $S_{4}$ & $S_{5}$ & $S_{6}$ & $S_{7}$ & $S_{8}$ & $S_{9}$ & $S_{10}$ & $S_{11}$ \\
\hline \multirow{4}{*}{$\begin{array}{c}V_{o 1}=0.5 * V_{s 1}+0.5 * V_{s 2} \text { and } V_{o 2}=0.5 * V_{s 1} \\
V_{o 1}=0.5 V_{s 1} \text { and } V_{o 2}=0.5 V_{s 1}+0.5 V_{s 2}\end{array}$} & $\phi_{1}$ & 1 & 0 & 0 & 0 & 0 & 0 & 0 & 1 & 1 & 0 & 0 \\
\hline & $\phi_{2}$ & 0 & 0 & 1 & 0 & 1 & 1 & 0 & 0 & 1 & 1 & 0 \\
\hline & $\phi_{3}$ & 0 & 1 & 0 & 0 & 0 & 0 & 0 & 1 & 1 & 0 & 0 \\
\hline & $\phi_{4}$ & 0 & 0 & 1 & 1 & 1 & 1 & 0 & 0 & 0 & 0 & 1 \\
\hline \multicolumn{13}{|c|}{ OTHER VCRs } \\
\hline$V o 1=V_{s 1}$ and $V_{o 2}=0.5 V_{s 1}$ & \multicolumn{6}{|c|}{$V_{o 2}=2 V_{s 1}$ and $V_{o 1}=1.5 V_{s 1}$} & \multicolumn{6}{|c|}{$V_{o 1}=0.5 V_{s 2}$ and $V_{o 2}=0.5 V_{s 1}+V_{s 2}$} \\
\hline$V_{o 2}=V_{s 2}$ and $V_{o 1}=0.5 V_{s 2}$ & \multirow{2}{*}{\multicolumn{6}{|c|}{$V_{o 1}=0.5 V_{s 1}+0.5 V_{s 2}$ and $V_{o 2}=0.5 V_{s 2}$}} & \multicolumn{6}{|c|}{$V_{o 1}=V_{s 2}$ and $V_{o 2}=0.5 V_{s 1}+V_{s 2}$} \\
\hline$V_{o 2}=V_{s 1}+V_{s 2}$ and $V_{o 1}=V_{s 1}$ & & & & & & $V_{o 1}=V_{s 1}$ and $V_{o 2}=0.5 V_{s 2}$ & \multicolumn{6}{|c|}{$V_{o 1}=2 V_{s 1}+2 V_{s 2}$ and $V_{o 2}=2 V_{s 1}$} \\
\hline$V_{o 1}=0.5 V_{s 2}$ and $V_{o 2}=0.5 V_{s 1}+0.5 V_{s 2}$ & \multicolumn{6}{|c|}{$V_{o 2}=V_{s 2}$ and $V_{o 1}=0.5 V_{s 1}$} & \multicolumn{6}{|c|}{$V_{o 2}=2 V_{s 1}+2 V_{s 2}$} \\
\hline$V_{o 1}=2 V_{s 1}$ and $V_{o 2}=1.5 V_{s 1}$ & \multirow{2}{*}{\multicolumn{6}{|c|}{$\begin{array}{c}V_{o 1}=0.5 V_{s 1} \text { and } V_{o 2}=0.5 V_{s 1}+V_{s 2} \\
V_{o 1}=V_{s 1} \text { and } V_{o 2}=V_{s 1}+0.5 V_{s 2}\end{array}$}} & \multicolumn{6}{|c|}{$V_{o 1}=0.5 V_{s 1}$ and $V_{o 2}=V_{s 1}$} \\
\hline$V_{o 2}=V_{s 2}$ and $V_{o 1}=0.5 V_{s 1}+V_{s 2}$ & & & & & & & & $=0.5$ & $s 1 \mathrm{a}$ & $V_{o}$ & $=0.5$ & $+V_{s 2}$ \\
\hline$V_{o 1}=V_{s 2}$ and $V_{o 2}=0.5 V_{s 2}$ & \multicolumn{6}{|c|}{$V_{o 2}=2 V_{s 2}$ and $V_{o 2}=1.5 V_{s 2}$} & \multicolumn{6}{|c|}{$V_{o 2}=V_{s 1}$ and $V_{o 1}=V_{s 1}+0.5 V_{s 2}$} \\
\hline$V_{o 2}=V_{s 1}$ and $V_{o 1}=0.5 V_{s 2}$ & \multicolumn{6}{|c|}{$V_{o 2}=2 V_{s 1}+2 V_{s 2}$ and $V_{o 1}=2 V_{s 1}$} & \multicolumn{6}{|c|}{$V_{o 1}=2 V_{s 1}+2 V_{s 2}$ and $V_{o 2}=2 V_{s 2}$} \\
\hline$V_{o 2}=V_{s 1}+V_{s 2}$ and $V_{o 1}=V_{s 2}$ & \multirow{2}{*}{\multicolumn{6}{|c|}{$\begin{array}{c}V_{o 1}=V_{s 2} \text { and } V_{o 2}=0.5 V_{s 1} \\
V\end{array}$}} & \multicolumn{6}{|c|}{$V_{o 1}=2 V_{s 2}$ and $V_{o 2}=1.5 V_{s 2}$} \\
\hline$V_{o 2}=0.5 V_{s 2}$ and $V_{o 1}=0.5 V_{s 2}+V_{s 1}$ & \multicolumn{5}{|c|}{$V_{o 1}=V_{s 1}+V_{s 2}$ and $V_{o 2}=V_{s 1}$} & & \multicolumn{6}{|c|}{$V_{o 1}=V_{s 1}+V_{s 2}$ and $V_{o 2}=V_{s 2}$} \\
\hline
\end{tabular}

For example VCR of $\left(V_{o 1}=0.5 * V_{s 1}+0.5 * V_{s 2}\right)$, and $V_{o 2}=0.5 * V_{s 1}$ is chosen. To configure the given SC converter VCR, 4 phases $\left(\phi_{1}, \phi_{2}, \phi_{3}, \phi_{4}\right.$ are required as shown in Fig 2. For $\phi_{1}$ and $\phi_{2}$ series charging and parallel discharging of flying capacitors is required which results in $V_{o 2}=0.5 * V_{s 1}$. In the $\phi_{3}$ capacitors are charged again, capacitor voltage level becames $0.5 * V_{s 2}$. Similarly for $\phi_{4}$ newly charged parallelly connected capacitors get in to series with $R_{o 2}$ which has already gained the voltage level of $0.5 * V_{s 1}$. Hence results $0.5 * V_{s 1}+0.5 * V_{s 2}$ across $R_{o 1}$. Switching pattern is given in Table I. Authors in [8], implemented 11 VCRs using 2

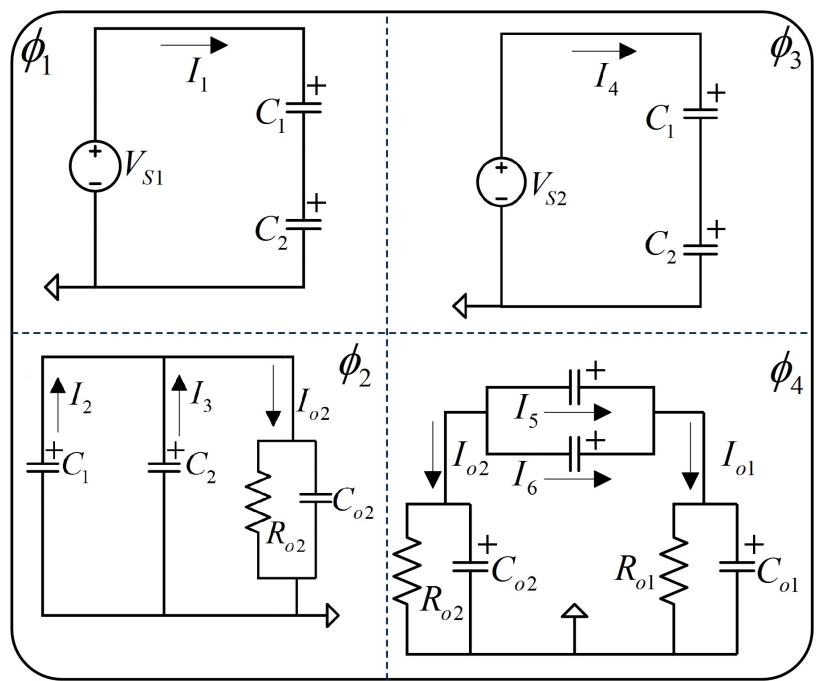

Fig. 2: Different phases of proposed converter $V_{o 1}=(0.5 *$ $\left.V_{s 1}\right)+\left(0.5 * V_{s 2}\right)$ and $V_{s 2}=0.5 * V_{s 1}$

capacitors and 9 switches and 5 diodes. This SC converter could implement summation of inputs as well. The limitation is that only one of the inputs can have non-unity gain. For example, circuit can be reconfigured for $V_{s 1}+0.5 * V_{s 2}$ or $V_{s 2}+0.5 * V_{s 2}$, but not $0.5 * V_{s 1}+0.5 * V_{s 2}$. So, the design proposed in this paper claims general advantages of dual output system, can also resolve VCRs which are impossible with single output.

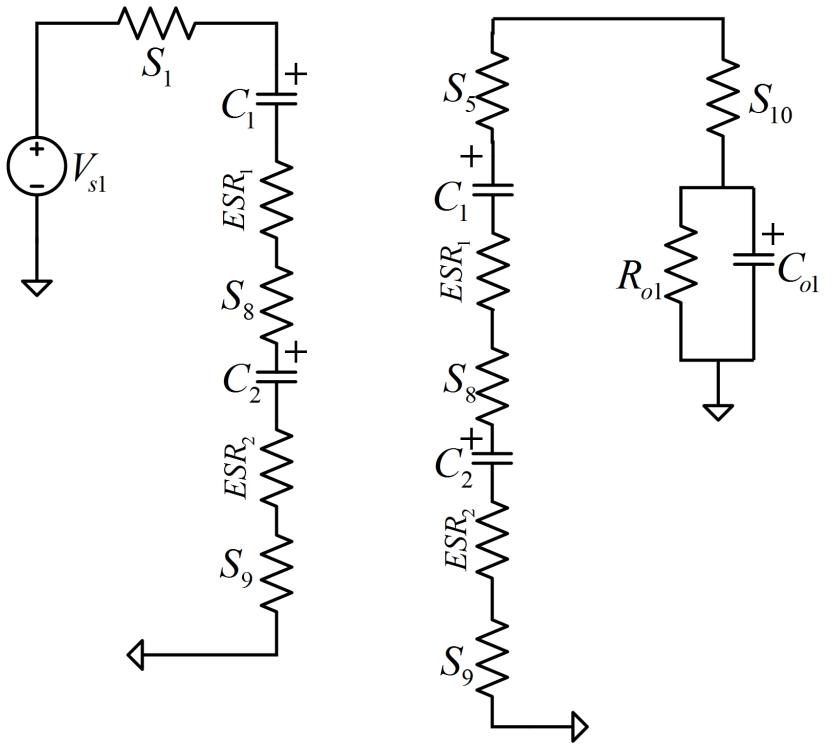

(a) Equivalent resistance of charging state and discharging state of $V_{o 1}$
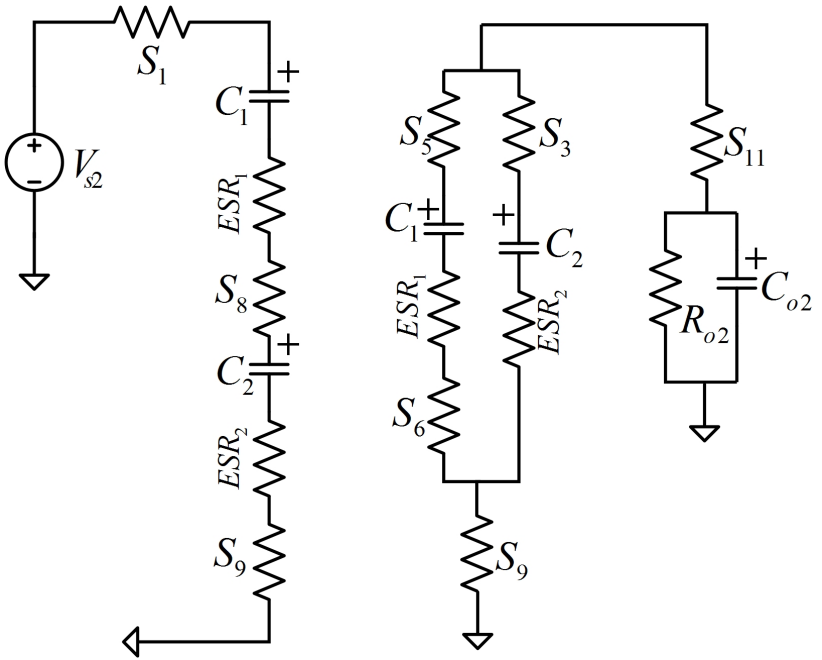

(b) Equivalent resistance of charging and discharging state of $V_{o 2}$

Fig. 3: Equivalent resistance of state $V_{o 1}=V_{s 1}$ and $V_{o 1}=0.5 * V_{s 2}$ 
TABLE II: Modelled and simulated value comparison of $V_{o 1}$ and $V_{o 2}$

\begin{tabular}{|c|c|c|c|c|c|c|c|c|}
\hline \multirow{2}{*}{$\begin{array}{c}\text { Frequency } \\
\text { kHz }\end{array}$} & \multicolumn{2}{|c|}{$V_{o 1}, I_{o 1}=25 \mathrm{~mA}$} & \multicolumn{2}{c|}{$V_{o 2}, I_{o 2}=12.5 \mathrm{~mA}$} & \multicolumn{2}{c|}{$\eta[\%]$} & \multicolumn{2}{c|}{$\eta[\%]$} \\
\cline { 2 - 9 } & Model [V] & Simulated [V] & Model [V] & Simulated [V] & Model & Simulated & Model & Simulated \\
\hline 100 & 4.68 & 4.77 & 2.43 & 2.41 & 93.6 & 95.4 & 97.2 & 96.4 \\
\hline 50 & 4.68 & 4.77 & 2.43 & 2.41 & 93.6 & 95.4 & 97.2 & 96.4 \\
\hline 25 & 4.68 & 4.75 & 2.43 & 2.41 & 93.6 & 95.0 & 97.2 & 96.4 \\
\hline
\end{tabular}

\section{ANALysis AND MODELING OF MULTiPle VCRS DUAL-INPUT AND DUAL OUTPUT SCC}

Equivalent resistance of the circuit is another important parameter of SC converter. Dual output systems commonly face with the issue known as a cross coupling effect, i.e, when changes in one output affects the second output [2]. Such kind of phenomenon can be detected by simulations or by analysis of switching pattern. If implementations of outputs are independent from each other, then two outputs can be assumed as decoupled and analyzed separately following the output analysis [8], [9] in a separate way. Two possible cases are discussed below and analysis are performed, (i.e). decoupled and cross coupled.

\section{A. Case 1: Decoupled}

For simplicity the VCR, $V_{o 1}=V_{s 1}$ and $V_{o 2}=0.5 * V_{s 1}$ is considered for analysis. Multiphase switching is used to generate four phases $(\phi)$ as shown in Fig 4. For both outputs

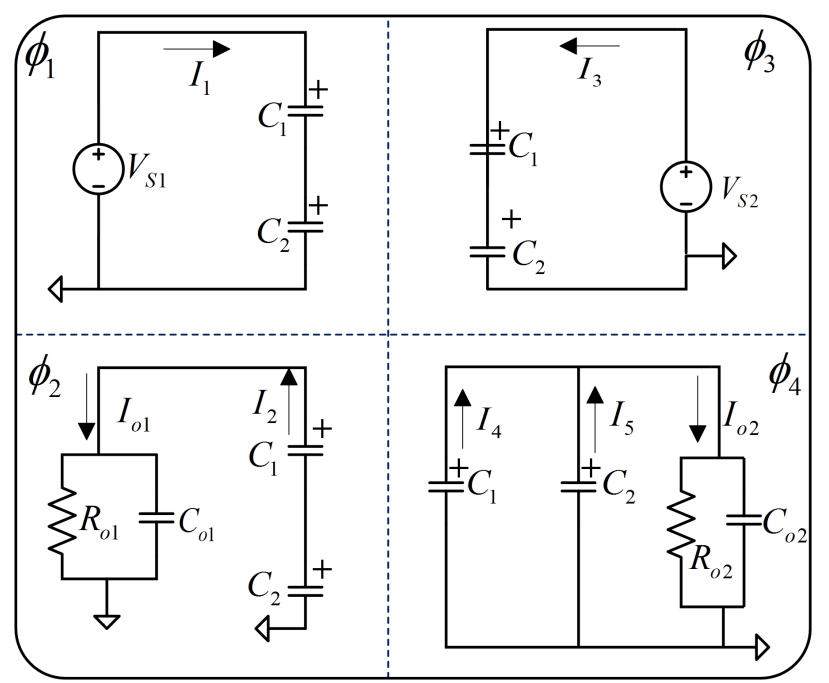

Fig. 4: Different phases of proposed converter $V_{o 1}=V_{s 1}$ and $V_{o 2}=0.5 * V_{s 2}$

charging and discharging are performed individually so that equivalent resistance can be obtained for each of the outputs independently. From Fig 4 it is clear that $\phi_{1}$ and $\phi_{2}$ are mainly responsible for the implementation of $V_{o 1}$ and $\phi_{3}$ and $\phi_{4}$ are responsible for implementing $V_{o 2}$. Following to the procedure described in [8], [9] charge balance and nodal KCL analysis
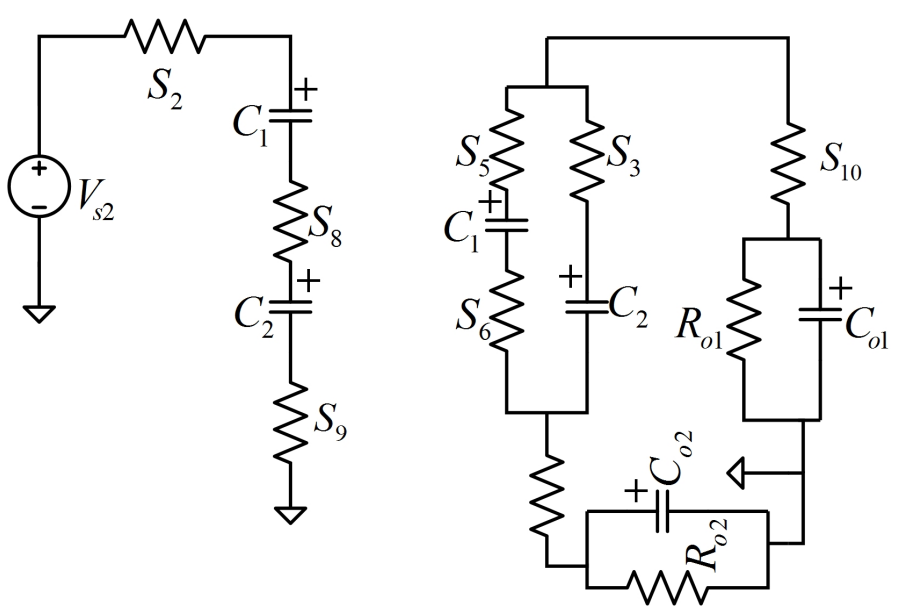

(a) Equivalent resistance of charging state and discharging state of $V_{o 1}$

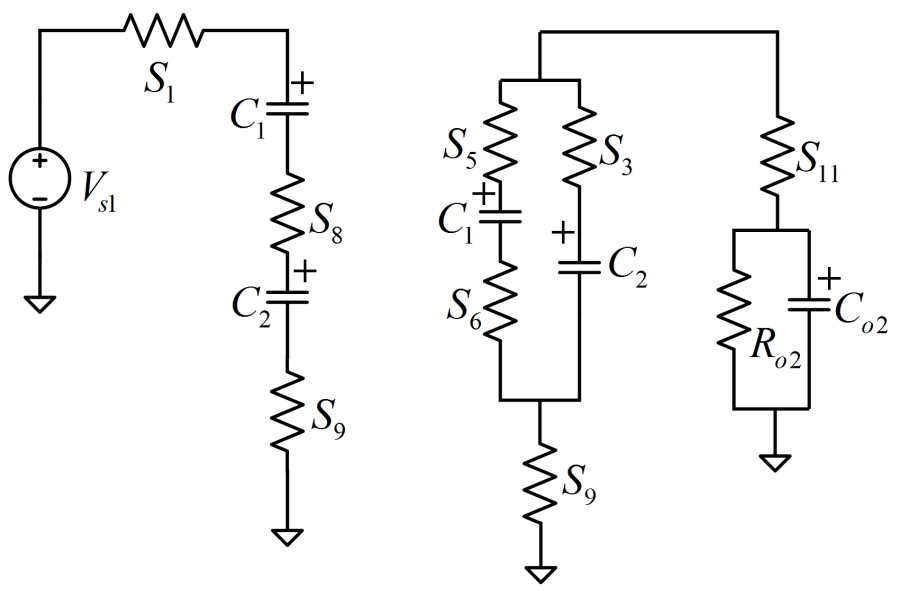

(b) Equivalent resistance of charging and discharging state of $V_{o 2}$

Fig. 5: Equivalent resistance of state $V_{o 1}=\left(0.5 * V_{s 1}\right)+\left(0.5 * V_{s 2}\right)$ and $V_{o 1}=0.5 * V_{s 1}$

for $\phi_{1}$ and $\phi_{2}$ yields following system of equations:

$$
\begin{array}{r}
I_{1}-I_{2}=0 \\
I_{1}=I_{0} \\
I_{1}=I_{2}=I_{0}
\end{array}
$$

Using the (1) equivalent resistance $\left(R_{e q 1}\right)$ [9], [2], [10] is given by:

$$
\begin{aligned}
R_{e q 1} & =\frac{1}{2 f_{s} * C_{e q 1}} \operatorname{coth}\left(\frac{1}{8 f_{s} * C_{e q 1} * r_{e q 1}}\right) \\
& +\frac{1}{2 f_{s} * C_{e q 2}} \operatorname{coth}\left(\frac{1}{8 f_{s} * C_{e q 2} * r_{e q 2}}\right)
\end{aligned}
$$


Using Fig 3a parameters of (2) is given in (3), where ESR is equivalent series resistance of capacitors

$$
\begin{array}{r}
r_{e q 1}=3 * r_{o n}+2 E S R \\
r_{e q 2}=4 * \text { ron }+2 E S R \\
C_{e q 1}=\frac{C}{2}=C_{e q 2}
\end{array}
$$

Similarly for $R_{e q 2}$, the equations are given by,

$$
\begin{aligned}
R_{e q 2}= & \frac{1}{8 f_{s} * C_{e q 1}} \operatorname{coth}\left(\frac{1}{8 f_{c 1} * C_{e q 1} * r_{e q 1}}\right) \\
& +\frac{1}{2 f_{s} * C_{e q 2}} \operatorname{coth}\left(\frac{1}{8 f_{s} * C_{e q 2} * r_{e q 2}}\right)
\end{aligned}
$$

$$
\begin{array}{r}
r_{e q 1}=3 * r_{o n}+2 E S R \\
r_{e q 2}=\frac{8}{3} r_{o n}+\frac{E S R}{2} \\
C_{e q 1}=\frac{C}{2} \\
C_{e q 1}=2 C
\end{array}
$$

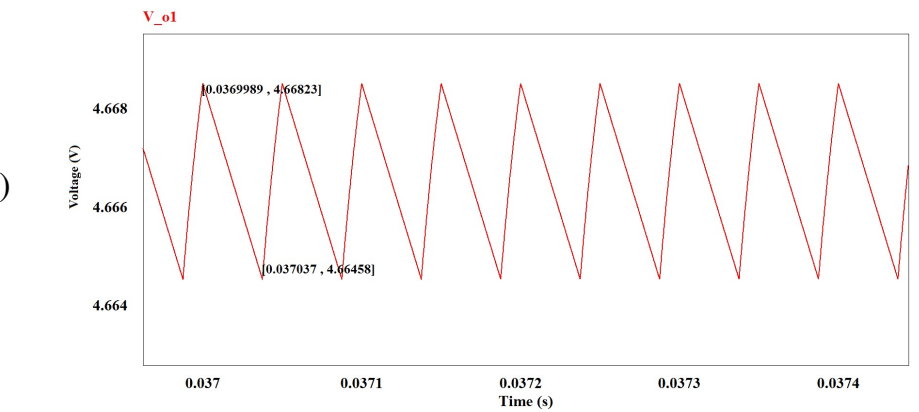

Assume, all switches $r_{\text {on }}$ are identical, capacitance and parasitic effects (ESR) are the same for both flying capacitors, filter capacitance $\left(C_{o 1}\right.$ and $\left.C_{o 2}\right)$ is large compared to flying capacitance $\left(C_{1}\right.$ and $\left.C_{2}\right)$ value. The equivalent resistance of the proposed converter output is given in (4) and (2). For all other VCRs given in Table I follows similar steps to find the $R_{o 1}$ and $R_{o 2}$ of proposed SC converter assuming no coupling effects. Simulations show that, there is some minor residual coupling between the outputs. By neglecting the coupling effects Table II is obtained using the methodology [8], [9].

\section{B. Case 2: coupled}

Consider the VCR $\left(V_{o 1}=\left(0.5 * V_{s 1}\right)+\left(0.5 * V_{s 2}\right)\right.$ and $V_{o 2}=$ $\left.0.5 * V_{s 1}\right)$. As it is discussed in Section II, implementation of this VCR requires two outputs being in the same phase as shown in Fig 5, which makes difficult to avoid cross coupling phenomenon. Modeling of the circuit based on two separate equivalent resistances may result in highly inaccurate results. Modelling and equivalent resistance of coupled dual output systems is major area of future studies. By now, verification of this kind of VCRs is limited to only simulations.

\section{Simulation Results and Discussion}

Simulation and parameter selection of proposed converter is discussed in this section. For simplicity, analysis is performed for only one $\operatorname{VCR}\left(V_{o 1}=\left(0.5 * V_{s 1}\right)+\left(0.5 * V_{s 2}\right)\right.$, $\left.V_{o 2}=0.5 * V_{s 1}\right)$ and $\left(V_{o 1}=V_{s 1}, V_{o 2}=0.5 * V_{s 2}\right)$. Real time parameters are considered for simulation and modeling the converter, details are as follows: MAX4678 CMOS analog switch parameter are used for switches $S_{1}-S_{11}$ referring to the Fig 1 with $r_{o n}=0.4 \Omega$. The flying capacitor and the output capacitor are rated by $22 \mu \mathrm{F}$ with ESR of 100 $\mathrm{m} \Omega, 220 \mu \mathrm{F}$ and the load resistance of $200 \Omega$ of the load current ranges from $25 \mathrm{~mA}$ to $100 \mathrm{~mA}$ at voltage range of (b) Output ripple of $V_{o 1}$

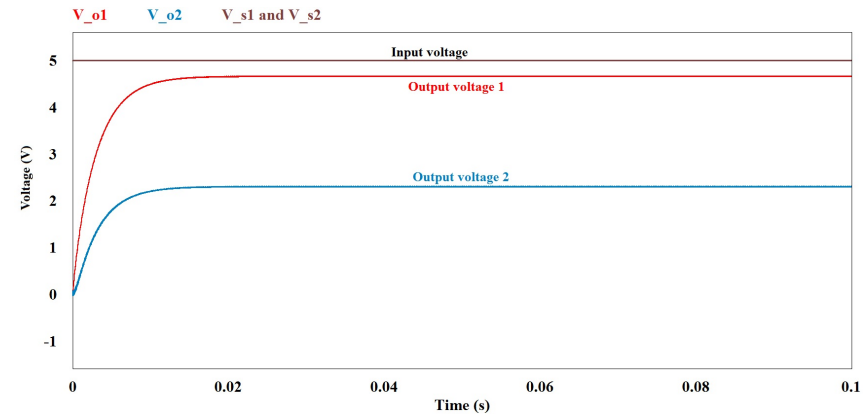

(a) Output voltage and input voltage

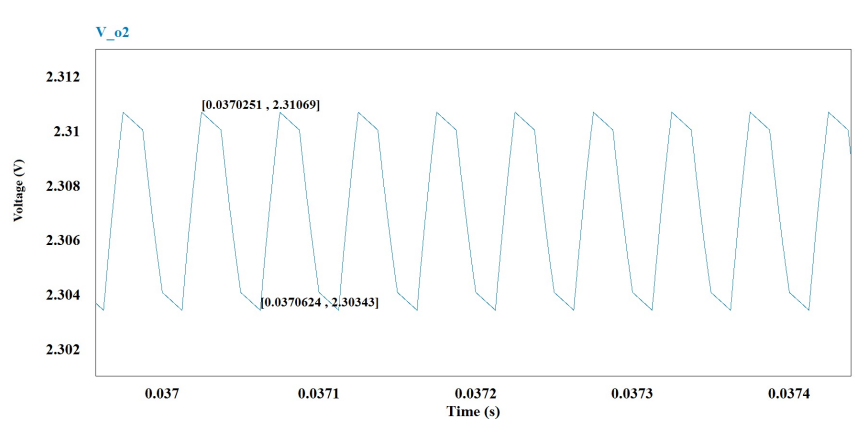

(c) Output ripple of $V_{o 2}$

Fig. 6: Simulation result VCR of $V_{o 1}=\left(0.5 * V_{s 1}\right)+\left(0.5 * V_{s 2}\right)$

\begin{tabular}{|c|c|c|c|c|}
\hline VCRs & $V_{s 1}$ & $V_{s 2}$ & $V_{o 1}$ & $V_{o 2}$ \\
\hline $\begin{array}{c}V_{o 1}=\left(0.5 * V_{s 1}\right)+\left(0.5 * V_{s 2}\right) \\
V_{o 2}=0.5 * V_{s 1}\end{array}$ & 4 & 6 & 4.985 & 1.991 \\
\hline $\begin{array}{c}V_{o 1}=\left(0.5 * V_{s 1}\right)+\left(V_{s 2}\right) \\
V_{o 2}=V_{s 2}\end{array}$ & 6 & 4 & 6.999 & 2.999 \\
\hline $\begin{array}{c}V_{o 1}=2 * V_{s 2} \\
V_{o 2}=1.5 * V_{s 2}\end{array}$ & 4 & - & 7.997 & 5.997 \\
\hline
\end{tabular}
and $V_{o 2}=0.5 * V_{s 1}$

$5 \mathrm{~V}-10 \mathrm{~V}$. Switching Frequency $\left(f_{s}\right)$ is varied accordingly to verify the proposed converter efficiency. For modelling the converter, equivalent resistance need to be calculated and it is verified by applying different frequency that are discussed in Table II. In addition, the results are verified for different VCR states as discussed in Table III. To verify the design,

TABLE III: Different VCRs simulated output

PSIM simulation tool was used. Simulation parameters strictly follow to the circuit ratings in this Section IV. Fig 6 illustrates 
TABLE IV: Comparison of previous SC VCRs

\begin{tabular}{|c|c|c|c|c|c|c|}
\hline Authors & Number of switches & No of input & No of output & No of capacitors & VCRs & Specification \\
\hline$[11]$ & 15 & 1 & 1 & 5 & 2 & $1 \mathrm{~V},<250 \mathrm{~mA}$ \\
\hline$[12],[13]$ & 4 & 2 & 1 & 2 & 1 & $36 \mathrm{~V},<120 \mathrm{~mA}$ \\
\hline$[5]$ & 7 & 1 & 1 & 3 & 2 & $5.4 \mathrm{~V},<120 \mathrm{~mA}$ \\
\hline$[4]$ & 14 (include diodes) & 2 & 1 & 3 & 11 & $9 \mathrm{~V},<120 \mathrm{~mA}$ \\
\hline This work & 11 & 2 & 2 & 3 & 32 & $10 \mathrm{~V},<100 \mathrm{~mA}$ \\
\hline
\end{tabular}

the output voltages and voltage ripples of proposed VCR $\left(V_{o 1}=\left(0.5 * V_{s 1}\right)+\left(0.5 * V_{s 2}\right)\right.$ and $\left.V_{o 2}=0.5 * V_{s 1}\right)$. Similarly for Fig 7 illustrates the output voltages and voltage ripples of VCR $\left(V_{o 1}=V_{s 1}\right.$ and $\left.V_{o 2}=0.5 * V_{s 2}\right)$. From Table II it is clear that modelled and simulated results are good agreement to verify the proposed converter topology. Table IV discusses about previous work done on different VCRs. We easily claim that the proposed converter is more advantageous over other $\mathrm{SC}$ converter. The main feature of the converter is VCRs can be generated independently for two different input and output voltages.

\section{CONCLUSIONS}

This work proposed the design for dual-input and dualoutput SCC. The converter uses two capacitors and 11 switches without any magnetic elements. The designed converter is analyzed and modeled for all $32 \mathrm{VCRs}$ by using equivalent resistance and verified by applying the same values in simulation. The simulation values and modeling values are in good agreement for a new converter design. The proposed design is mainly advantageous for power management ICs. Future scope of this work is implementation of negative VCRs and other fractional implementations such as 1:3, 2:3, 3:4. Moreover, analysis of coupling effects are needed to be studied.

\section{REFERENCES}

[1] V. Subburaj and D. Jena, "Sub-period interleaved fibonacci switched capacitor converter," in Region 10 Conference (TENCON), 2016 IEEE. IEEE, 2016, pp. 2892-2895.

[2] A. Zhaikhan, A. Daulbayeva, A. Berdygozhin, and A. Ruderman, "Novel voltage target ratios and minimal norm principle for reconfigurable multiphase single-and dual-output switched capacitor converters," in Power Electronics, Machines and Drives (PEMD 2016), 8th IET International Conference on. IET, 2016, pp. 1-8.

[3] V. Subburaj, D. Jena, R. Kumar, A. V. Deshmukh, B. Nayak, and H. Bansal, "Design of series, $\mathrm{f} i=\mathrm{f} i-1+\mathrm{f} i-3$ for the denominators $(1$, 2, 6) of switched capacitor converter," in Power Electronics, Intelligent Control and Energy Systems (ICPEICES), IEEE International Conference on. IEEE, 2016, pp. 1-5.

[4] C. Abraham, B. R. Jose, J. Mathew, and M. Evzelman, "Modelling, simulation and experimental investigation of a new two input, seriesparallel switched capacitor converter," IET Power Electronics, vol. 10, no. 3, pp. 368-376, 2017.

[5] H.-E. W. L. C. Pumps, http://pdfserv.maxim-ic.com/en/ds/MAXIM1910MAXIM1912.pdf, 2014.

[6] H. E. S.-D. S. V. Converter, http://www.ti.com/lit/ds/symlink/lm2788.pdf, 2014.

[7] P. management IC-TPS65913, http://www.ti.com/product/TPS65913/, 2015.

[8] M. Evzelman and S. Ben-Yaakov, "Average-current-based conduction losses model of switched capacitor converters," IEEE Transactions on Power Electronics, vol. 28, no. 7, pp. 3341-3352, 2013.

[9] S. Ben-Yaakov, "On the influence of switch resistances on switchedcapacitor converter losses," IEEE Transactions on Industrial Electronics, vol. 59, no. 1, pp. 638-640, 2012.

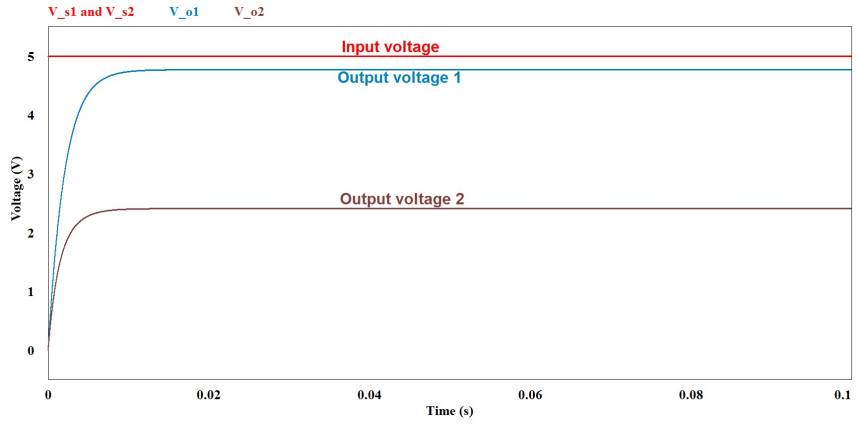

(a) Output voltage and input voltage

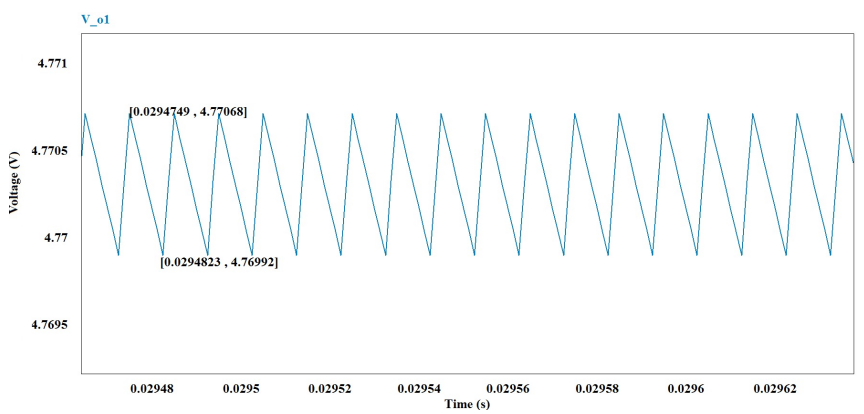

(b) Output ripple of $V_{o 1}$

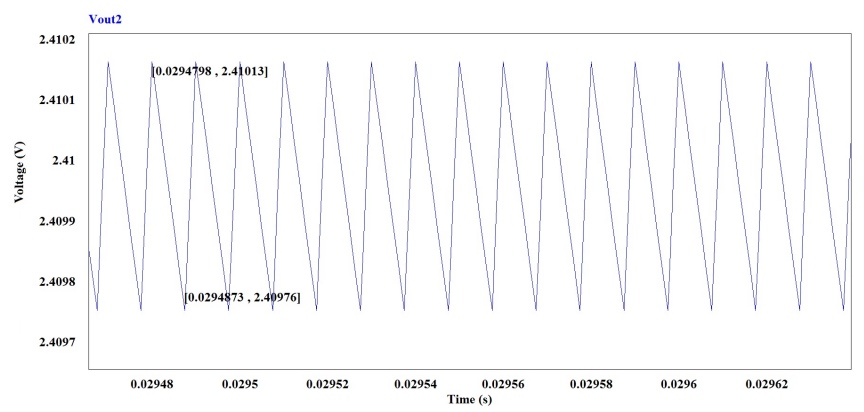

(c) Output ripple of $V_{o 2}$

Fig. 7: Simulation result VCR of $V_{o 1}=V_{s 1}$ and $V_{o 2}=0.5 * V_{s 2}$

[10] A. Kushnerov, "High-efficiency self-adjusting switched capacitor dc-dc converter with binary resolution," arXiv preprint arXiv:1003.4301, 2010.

[11] H. P. Le, Design techniques for fully integrated switched-capacitor voltage regulators. $\mathrm{PhD}$ thesis, University of California, Berkeley, 2015.

[12] Y. Yuan-mao and K. W. E. Cheng, "Multi-input voltage-summation converter based on switched-capacitor," IET Power Electronics, vol. 6, no. 9, pp. 1909-1916, 2013.

[13] Y. Yuanmao and K. Cheng, "Level-shifting multiple-input switchedcapacitor voltage copier," IEEE transactions on power electronics, vol. 27, no. 2, pp. 828-837, 2012. 